\title{
EARLY RESULTS FROM THE COSMIC BACKGROUND EXPLORER (COBE)
}

J. C. MATHER, M. G. HAUSER, C. L. BENNETT, N. W. BOGGESS, E. S. CHENG, R. E. EPLEE, JR., H. T. FREUDENREICH, R. B. ISAACMAN, T. KELSALL, C. M. LISSE, S. H. MOSELEY, JR., R. A. SHAFER, R. F. SILVERBERG, W. J. SPIESMAN, G. N. TOLLER, J. L. WEILAND

Laboratory for Astronomy and Solar Physics, Goddard Space Flight Center,

Greenbelt, $M D 20771$

S. GULKIS, M. JANSSEN

Jet Propulsion Laboratory, MS 169-506, 4800 Oak Grove Drive, Pasadena, CA 91109

P. M. LUBIN

UCSB, Dept. of Physics, Goleta, CA 93106

S. S. MEYER, R. WEISS

Department of Physics, Massachusetts Institute of Technology, Cambridge, MA 02139

\section{T. L. MURDOCK}

General Research Corporation, 5 Cherry Hill Drive, Suite 220, Danvers, MA 01923

G. F. SMOOT

Lawrence Berkeley Laboratory, 50-232, University of California, Berkeley, CA 94720

D. T. WILKINSON

Department of Physics, Jadwin Hall, Box 708, Princeton University, Princeton, NJ 08544

and

E. L. WRIGHT

UCLA Dept. of Astronomy, Los Angeles, CA 90024-1562

\begin{abstract}
The Cosmic Background Explorer, launched November 18, 1989, has nearly completed its first full mapping of the sky with all three of its instruments: a Far Infrared Absolute Spectrophotometer (FIRAS) covering 0.1 to $10 \mathrm{~mm}$, a set of Differential Microwave Radiometers (DMR) operating at $3.3,5.7$, and $9.6 \mathrm{~mm}$, and a Diffuse Infrared Background Experiment (DIRBE) spanning 1 to $300 \mu \mathrm{m}$ in ten bands. A preliminary map of the sky derived from DIRBE data is presented. Initial cosmological implications include: a limit on the Comptonization $y$ parameter of $10^{-3}$, on the chemical potential $\mu$ parameter of $10^{-2}$, a strong limit on the existence of a hot smooth intergalactic medium, and a confirmation that the dipole anisotropy has the spectrum expected from a Doppler shift of a blackbody. There are no significant anisotropies in the microwave sky detected, other than from our own galaxy and a $\cos \theta$ dipole anisotropy whose amplitude and direction agree with previous data. At shorter wavelengths, the sky spectrum and anisotropies are dominated by emission from 'local' sources of emission within our Galaxy and Solar
\end{abstract}

Y. Kondo (ed.), Observatories in Earth Orbit and Beyond, 9-18.

(C) 1990 Kluwer Academic Publishers. Printed in The Netherlands. 
System. Preliminary comparison of IRAS and DIRBE sky brightnesses toward the ecliptic poles shows the IRAS values to be significantly higher than found by $D I R B E$ at $100 \mu \mathrm{m}$. We suggest the presence of gain and zero-point errors in the IRAS total brightness data. The spacecraft, instrument designs, and data reduction methods are described.

\section{Introduction}

The cosmic microwave background radiation (CMBR) from the Big Bang, and the infrared background radiation from the first objects to form after the Big Bang, are the subjects of NASA's first dedicated cosmological satellite, the COBE. The mission has been described by Mather (1982) and Gulkis et al. (1990). Theoretical estimates for various distortions of the microwave and infrared background radiation spectra have been given by Bond, Carr, and Hogan (1986) and by many others in this very active field.

We summarize here the key events in the expanding universe which influence these backgrounds. Processes in the early expansion of the universe such as inflation and subsequent nucleosynthesis, which take place within the first few minutes after the initial explosion, set the scale for the largest anisotropies and inhomogeneities. The number of quanta in the CMBR is essentially fixed when the time scales for their creation and destruction exceed the expansion time scale, which occurs at a redshift $z \sim 10^{6}$ when photons outnumber matter particles by a factor of $\sim 10^{8}$. After this epoch the spectrum of the CMBR can be modified to a Bose-Einstein distribution with a chemical potential $\mu$ by such processes as black hole formation, cosmic string annihilation, matter/antimatter annihilation, or the decay of exotic particles. After a redshift of $z \sim 3 \times 10^{4}$, the process to re-establish equilibrium becomes too slow to achieve a Bose-Einstein distribution, and any other energy release into the radiation field mediated by hot electrons produces a mixture of blackbodies at different temperatures, characterized by the parameter $y$. After the decoupling of matter and radiation at $z \sim 10^{3}$, the photon field is free to move unimpeded, and the major features of the spectrum and angular distribution of the CMBR as now observed should have been established.

The processes leading to the formation of galaxies and large scale structures, as well as the epoch when these form, are major unknowns in current cosmology. One can bracket the epoch by noting that the earliest quasars have a $z<5$ and that galaxies at their present size would overlap at $z$ between 20 and 100. The formation of galaxies may well be accompanied by the creation of a hot intergalactic medium, which could produce anisotropies and spectral distortions in the CMBR as well as a signature in the infrared. Furthermore, it is expected that precursors to the large scale structures should have left anisotropies and possibly spectral distortions in the CMBR.

As illustrated in Fig. 1, local astrophysical sources make it difficult to measure these traces of the pregalactic universe. Interplanetary dust reflects and absorbs sunlight and reradiates at a temperature of about $250 \mathrm{~K}$, while interstellar dust reflects and absorbs starlight and reradiates at a temperature of about $25 \mathrm{~K}$. At longer wavelengths interstellar electrons produce synchrotron and free-free emission. The $C O B E$ mission was designed to produce a complete data set that would permit 


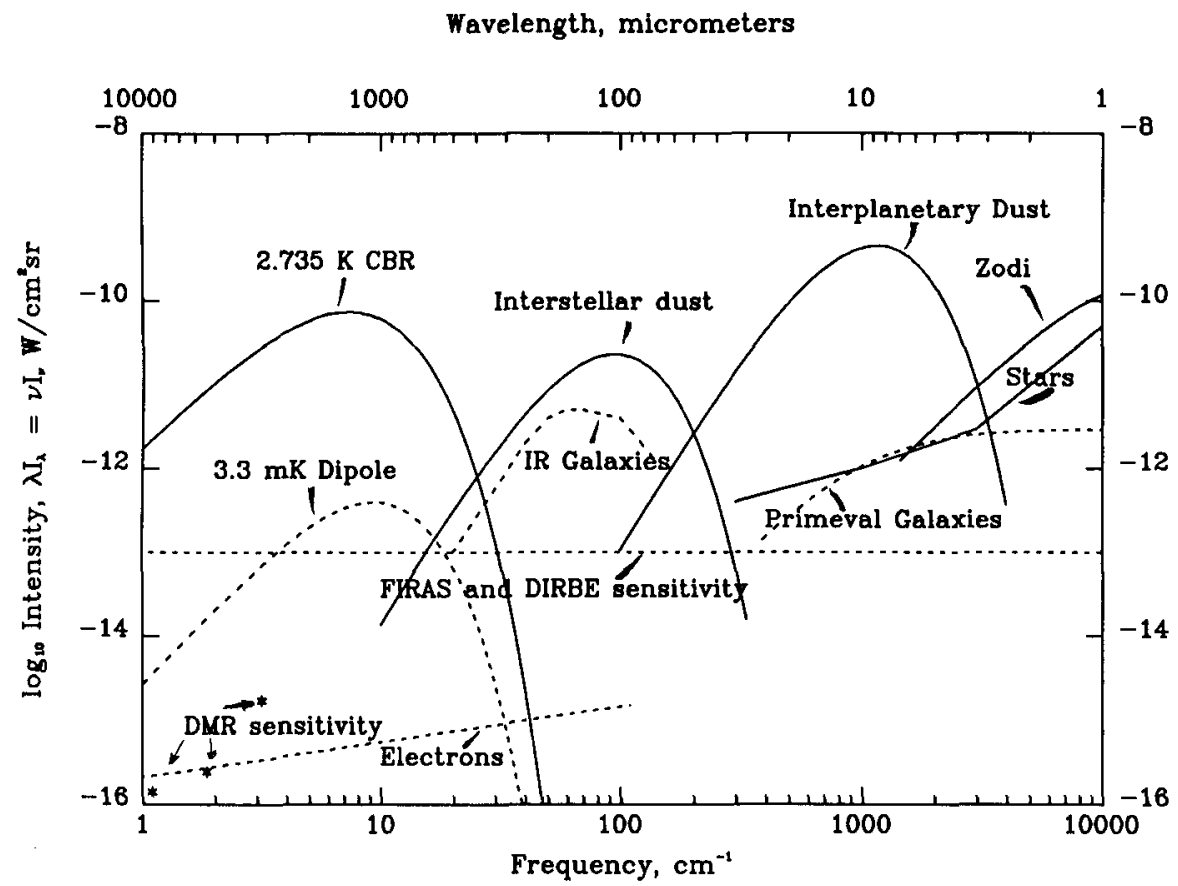

Fig. 1. Schematic of diffuse radiation fields observable by the COBE. Except for the CBR and dipole, these curves are not based on COBE measurements, which are still being analyzed.

detailed models of these local sources, and a subtraction of them to reveal the true cosmological background. The $C O B E$ sensitivities per beamwidth and spectral element are also shown on Fig. 1, and are well below the limitations set by local astrophysics over most of the bands.

\section{Spacecraft and Orbit}

The COBE was launched by NASA on a Delta rocket on November 18, 1989 from the Western Space and Missile Center at Lompoc, California into a nearly polar orbit over the terminator region of the Earth. With an altitude of $900 \mathrm{~km}$ and an orbital inclination of $99^{\circ}$, the plane of the orbit precesses at one revolution per year, chosen to follow the apparent motion of the Sun and keep the orbit over the terminator. In this orbit, the spacecraft can be oriented to point away from the Earth and approximately perpendicular (control range is $90^{\circ}$ to $98^{\circ}$ ) to the Sun for the duration of the mission. For two months around the summer solstice, the satellite passes through the Earth's shadow near the South Pole for up to 18 minutes in each 103 minute orbit, and for a similar period near the North Pole the Earth limb can rise a few degrees above the top of the instrument shield and illuminate 
the instruments.

The three instruments are all located inside a deployable shield in the top half of the spacecraft, whose upper edge after deployment is roughly coplanar with the instrument apertures. The shield protects them from heat and light from the Earth and Sun, from terrestrial radars, and from the spacecraft telemetry antenna located at the bottom of the COBE. The inner surface of the shield is covered with multilayer insulation and reaches a temperature of $150 \mathrm{~K}$. The FIRAS and $D I R B E$ instruments are located inside a helium cryostat in the center of the shield, and the DMR receivers are located in three boxes mounted around the outside of the cryostat. The cryostat is similar to that used on the Infrared Astronomical Satellite (IRAS) and carried 600 liters of superfluid helium at launch, sufficient to last for one year after launch. The temperature of the helium in orbit is $1.4 \mathrm{~K}$, while the FIRAS and DIRBE instruments are heated to $1.5 \mathrm{~K}$ by their cryogenic power dissipation of $22 \mathrm{~mW}$. The helium evaporates through a porous plug which separates the liquid and gas phases, and the efflux gas exits through a vent oriented towards the Earth along the spacecraft spin axis. Because the axis is about $97^{\circ}$ from the velocity vector, there is a component of the thrust from the helium vent along the orbit vector which lowers the orbit by $\sim 30 \mathrm{~m} /$ day.

The spacecraft spins at $0.8 \mathrm{rpm}$ about its symmetry axis, to scan the $D M R$ and $D I R B E$ beams rapidly around the sky. Attitude control is provided by two large counter-rotating momentum wheels to create the spin, and three small momentum wheels with axes perpendicular to the spacecraft axis for three axis fine control. Sun and Earth sensors provide basic aspect information $\left(3 \sigma<1^{\circ}\right)$ and electromagnetic control bars exert torque against the Earth's magnetic field to keep the net angular momentum near zero. Gyroscopes provide fine resolution aspect information and are used by the attitude control servos for rate damping. Star sightings by the DIRBE instrument are used to calculate fine aspect solutions good to a few arcminutes. The spacecraft is powered by three solar cell panels which provide over 1000 watts of power in full sun, and $\mathrm{Ni}-\mathrm{Cd}$ storage batteries provide power during the shadow passages. Data are stored on two tape recorders and played back daily to a ground station at the Wallops Flight Facility in Virginia.

Commands are loaded to the onboard memory daily through the Tracking and Data Relay Satellite, and correct operations are verified 7 times per day through the same system.

\section{FIRAS}

The purpose of the FIRAS is to compare the spectrum of the CMBR with that of a precise blackbody, to enable the measurement of small deviations from a Planckian spectrum.

The FIRAS instrument covers two wavelength ranges, from 0.1 to $0.5 \mathrm{~mm}$ and from 0.5 to $10 \mathrm{~mm}$. It has a $7^{\circ}$ diameter beamwidth, established by a non-imaging parabolic concentrator, which has a flared aperture to reduce diffractive sidelobe responses. The instrument is calibrated by a full beam, temperature-controlled external blackbody, which can be moved into the beam by command. The FIRAS is the first instrument to measure the background radiation and compare it to such an 
accurate external full beam calibrator in flight. The spectral resolution is obtained with a polarizing Michelson interferometer, with separated input and output beams to permit fully symmetrical differential operation. One input beam is the sky or full aperture calibrator while the second input beam is provided by an internal temperature controlled reference blackbody, with its own parabolic concentrator. Both input concentrators and both calibrators are temperature controlled and can be set by command to any temperature between 2 and $25 \mathrm{~K}$. The standard operating condition is for the two concentrators and the internal reference body to be commanded to match the sky temperature, thereby yielding a nearly nulled interferogram which reduces almost all instrumental errors to negligible values.

The instrument's spectral resolution is limited by the maximum stroke length to $0.2 \mathrm{~cm}^{-1}$ in the long wavelength channels, and by the microprocessor buffer size and telemetry to $0.2 \mathrm{~cm}^{-1}$ in the short wavelength channel. The rms sensitivity is $\nu I_{\nu}$ $<10^{-9} \mathrm{~W} / \mathrm{m}^{2}$ sr per $7^{\circ}$ field and per $5 \%$ spectral resolution element after one year of operation. There are four large area $\left(0.5 \mathrm{~cm}^{2}\right)$ composite bolometer detectors, two on each output of the spectrometer. The separation of long and short wavelengths is accomplished by a capacitive grid dichroic filter.

The external calibrator determines the accuracy of the instrument. It is a reentrant cone shaped like a trumpet mute, made of Eccosorb CR-110 iron-loaded epoxy. The angles at the point and groove are $25^{\circ}$, so that a ray reaching the detector has suffered 7 specular reflections from the calibrator. The calculated reflectance for this design, including diffraction and surface imperfections, is less than $10^{-3}$ from 0.5 to $5 \mathrm{~mm}$. The instrument is calibrated by measuring spectra with the calibrator and all the other controllable sources within the instrument held at a sequence of different temperatures. The overall responsivity and the emissivity of each source can be determined relative to the external calibrator by solving a set of coupled linear equations.

The preliminary results of the FIRAS have been given by Mather et al. (1990) and may be summarized as follows. The intensity of the background sky radiation is consistent with a blackbody at $2.735 \pm 0.06 \mathrm{~K}$, and deviations from this blackbody at the spectral resolution of the instrument are less than $1 \%$ of the peak brightness. The quoted uncertainty in temperature is due an uncertainty in the thermometer calibration, and we expect to further refine the calibration by additional tests. The deviations can be fitted to the Sunyaev-Zel'dovitch form for Comptonization, giving a limit on the $y$ parameter of $|y|<10^{-3}(3 \sigma)$. They can also be fitted to a BoseEinstein distribution with a chemical potential, giving a limit of $|\mu|<10^{-2}(3 \sigma)$. There is a strong limit on the existence of a smooth hot intergalactic medium; it can contribute less than $3 \%$ of the $\mathrm{X}$-ray background radiation even at a reheating time as recent as $z=2$. There is no evidence of a distortion of the spectrum, such as that reported by Matsumoto et al. (1988a), and the measured temperature is consistent with previous reports.

The variation of the spectrum with position in the sky as measured by the FIRAS is dominated by the dipole pattern of the Doppler shift of the background temperature, plus a difference in the interstellar dust emission. A preliminary analysis of three weeks of data taken near the Galactic plane shows exactly such a variation. A more precise determination of the spectrum of the dipole was made 
by calculating the average spectra in two large circular regions of the sky each of angular diameter $60^{\circ}$, one centered at $(\alpha, \delta)=\left(11^{h} .1,-6^{\circ} .3\right)$ and the other at $\left(23^{h} .1,6^{\circ} .3\right)$, which lie along opposite ends of the dipole axis. The difference between these spectra can be fit extremely well by the difference of two blackbodies, and is consistent with a peak dipole amplitude of $3.3 \pm 0.3 \mathrm{mK}$ and the assumed dipole direction.

\section{DMR}

The Differential Microwave Radiometers are designed to measure the anisotropy of the cosmic background radiation at a set of wavelengths optimized to separate Galactic emissions from the CMBR. The DMR includes two radiometers at each of 3 frequencies $(31.5,53$, and $90 \mathrm{GHz}$, or $9.6,5.7$ and $3.3 \mathrm{~mm}$ wavelength). The receivers are standard two-horn Dicke-switched mixer-preamplifier devices, with ferrite switches operated at $100 \mathrm{~Hz}$. The IF bandwidths are $0.5,0.8$, and $0.8 \mathrm{GHz}$ respectively, and the radiometer sensitivities are $43,42,15,16,28$, and $19 \mathrm{mK}$ $\sec ^{1 / 2}$ for the six receivers. The design has been described by Smoot et al. (1990) and the calibration by Bennett et al. (1991).

Two corrugated-horn antennas feed each receiver. Each horn defines a $7^{\circ} \mathrm{FWHM}$ Gaussian beam, and the horns are oriented $30^{\circ}$ from the spin axis of the spacecraft and $60^{\circ}$ from each other. As the spacecraft spins and pitches in its orbit, and the orbit precesses during the year, all possible pairs of pixels on the sky $60^{\circ}$ apart are compared. A least-squares fit to these differences is made to recover a relative map of the sky; the DC term is lost in the differential measurement. In addition, the least square solution is used to estimate or place limits on certain potential sources of systematic errors. Beam switching between two non-fixed points on the sky gives twice as much effective observing time as a comparable single horn receiver switching between a fixed reference and the sky.

The receivers operate within protective thermal enclosures and RF shields to provide stable operating conditions. No evidence has yet been uncovered to indicate effects due to RF interference from ground radars, other satellites, or the COBE transmitter. The thermal stability of the instrument is excellent $(<0.01 \mathrm{~K} /$ day drift in physical temperature, $<0.001 \mathrm{~K} /$ day drift in instrument offset). The 53 and $90 \mathrm{GHz}$ receivers operate at $140 \mathrm{~K}$ for reduced detector noise, and the $31.5 \mathrm{GHz}$ receivers operate at $300 \mathrm{~K}$.

Preliminary maps of the sky made with all 6 receivers show that the expected receiver sensitivity has been achieved. The maps represent 120 days of observation, and over the two years of operations we expect more than a factor of two improvement in the random noise. No obvious structures are visible in the maps other than the expected Galactic plane emissions, and some small regions which included the moon (these will be removed in subsequent analysis). The Galactic sources show the expected variation in intensity with frequency. Analysis is underway to quantify the limits that can be placed on anisotropies and Galactic power law indices. The preliminary data are consistent with previous values from balloon and satellite work. We find the amplitude and direction of the dipole as $3.3 \pm 0.2 \mathrm{mK}$ towards $(\alpha, \delta)=\left(11^{h} .2 \pm 0 .{ }^{h} 2,-6^{\circ} \pm 2^{\circ}\right)$ expressed as 
equivalent thermodynamic temperature variation of a blackbody spectrum. All three DMR frequencies and the FIRAS results are consistent with the Doppler shift interpretation of the dipole anisotropy.

\section{DIRBE}

The purpose of the Diffuse Infrared Background Experiment is to conduct a definitive search for an isotropir cosmic infrared background radiation. Such a background is expected to be prenced by the cumulative emissions of pregalactic, protogalactic, and galactic systems. The experiment approach is to obtain absolute brightness maps of the full sky in 10 photometric bands $(\mathrm{J}, \mathrm{K}, \mathrm{L}$, and $\mathrm{M}$; the four IRAS bands at $12,25,60$, and $100 \mu \mathrm{m}$, and $120-200$ and $200-300 \mu \mathrm{m}$ bands). In order to facilitate discrimination of the contribution from the bright foreground due to scattering and emission from interplanetary dust, linear polarization is also measured in the $\mathrm{J}, \mathrm{K}$, and $\mathrm{L}$ bands, and all celestial directions are observed hundreds of times at all accessible solar elongation angles (depending upon ecliptic latitude) in the range $64^{\circ}$ to $124^{\circ}$.

The DIRBE instrument is an absolute radiometer, utilizing an off-axis folded Gregorian telescope with a $19 \mathrm{~cm}$ diameter primary mirror. The optical configuration is carefully designed for stray light immunity, utilizing both a secondary field stop and a Lyot stop, super-polished primary and secondary mirrors, a reflective forebaffle, extensive internal baffling, and a complete light-tight enclosure of the instrument. The instrument measures the absolute sky brightness by chopping between the sky and a zero-flux internal reference at $32 \mathrm{~Hz}$ using a tuning fork chopper. Instrumental offsets can be measured by closing a cold shutter located at the prime focus. All spectral bands view the same instantaneous fieldof-view, $0.7^{\circ} \times 0.7^{\circ}$, oriented at $30^{\circ}$ from the $C O B E$ spin axis. This allows the DIRBE to modulate solar elongation angles by $60^{\circ}$ during each rotation, and to sample fully $50 \%$ of the celestial sphere each day. A highly-reproducible internal reference source can stimulate all detectors when the shutter is closed to monitor the stability of instrument response.

At the present time, the DIRBE has mapped the full celestial sphere, though all available elongations will not be covered until the end of May. The data are of very high quality, showing good sensitivity, stability, and linearity, with few artifacts other than those induced by energetic particles in the South Atlantic Anomaly. Data processing to date has been limited to quick-look checking of daily data dumps. This checking includes creation of daily sky maps. Figure 2 shows an image created by combining $\mathbf{J}, \mathrm{K}$, and L-band data from several weeks during the first 4 months of the mission. At these wavelengths the sky is dominated by Galactic starlight, with a lesser contribution from zodiacal scattered light.

To meet the cosmological objective of the DIRBE, the foreground light from interplanetary and galactic sources must be discriminated from the total observed sky brightness. This task will require extensive careful modelling, which is just beginning. A conservative upper limit on extragalactic light is the total observed brightness in a relatively dark direction, such as the ecliptic poles. Figure 3 presents the infrared sky brightness toward the south ecliptic pole in all 10 DIRBE bands 


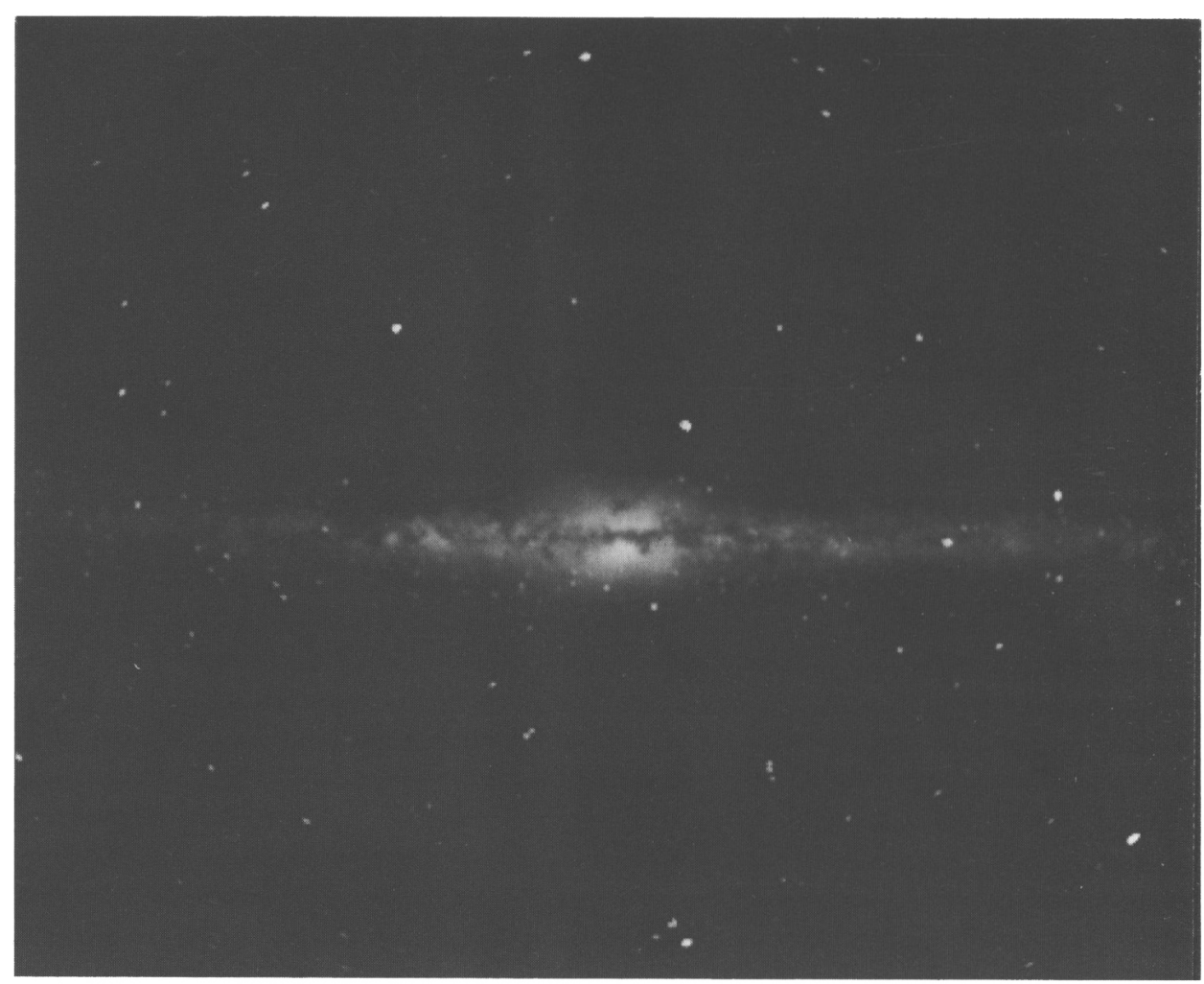

Fig. 2. DIRBE map of $90^{\circ}$ sector of the Galaxy, combining J, K, and L bands.

based upon a preliminary calibration. As expected, the faintest foregrounds occur at $3.4 \mu \mathrm{m}$ (L-band), in the minimum between interplanetary dust scattering of sunlight and re-emission of absorbed sunlight by the same dust, and longward of $100 \mu \mathrm{m}$, where interstellar dust emission begins to decrease. Through careful modelling, we hope to be able to discriminate isotropic residuals at a level as small as a percent of the foregrounds. These near-infrared and submillimeter 'windows' will allow the most sensitive search for the elusive cosmic infrared background.

The DIRBE total brightnesses at $\mathrm{J}, \mathrm{K}, \mathrm{L}$, and $\mathrm{M}$ band are similar to those reported by Matsumoto et al. (1988b), though quantitative statements must await comparison of observations made in the same direction on the same day number of the year (to get the same interplanetary dust contribution). A similar conclusion is reached when our data at 100,150 , and $240 \mu \mathrm{m}$ are compared with the submillimeter data of Matsumoto et al. (1988a).

We have made a more detailed comparison at several points on the sky with IRAS total brightness observations at $12,25,60$, and $100 \mu \mathrm{m}$. By using several points, we can distinguish zero-point offsets and gain differences. We find evidence for zero-point differences, evidently not constant in time, of a few

$\mathrm{MJy} / \mathrm{sr}$ at all of these wavelengths. These are most significant (as a fraction of 


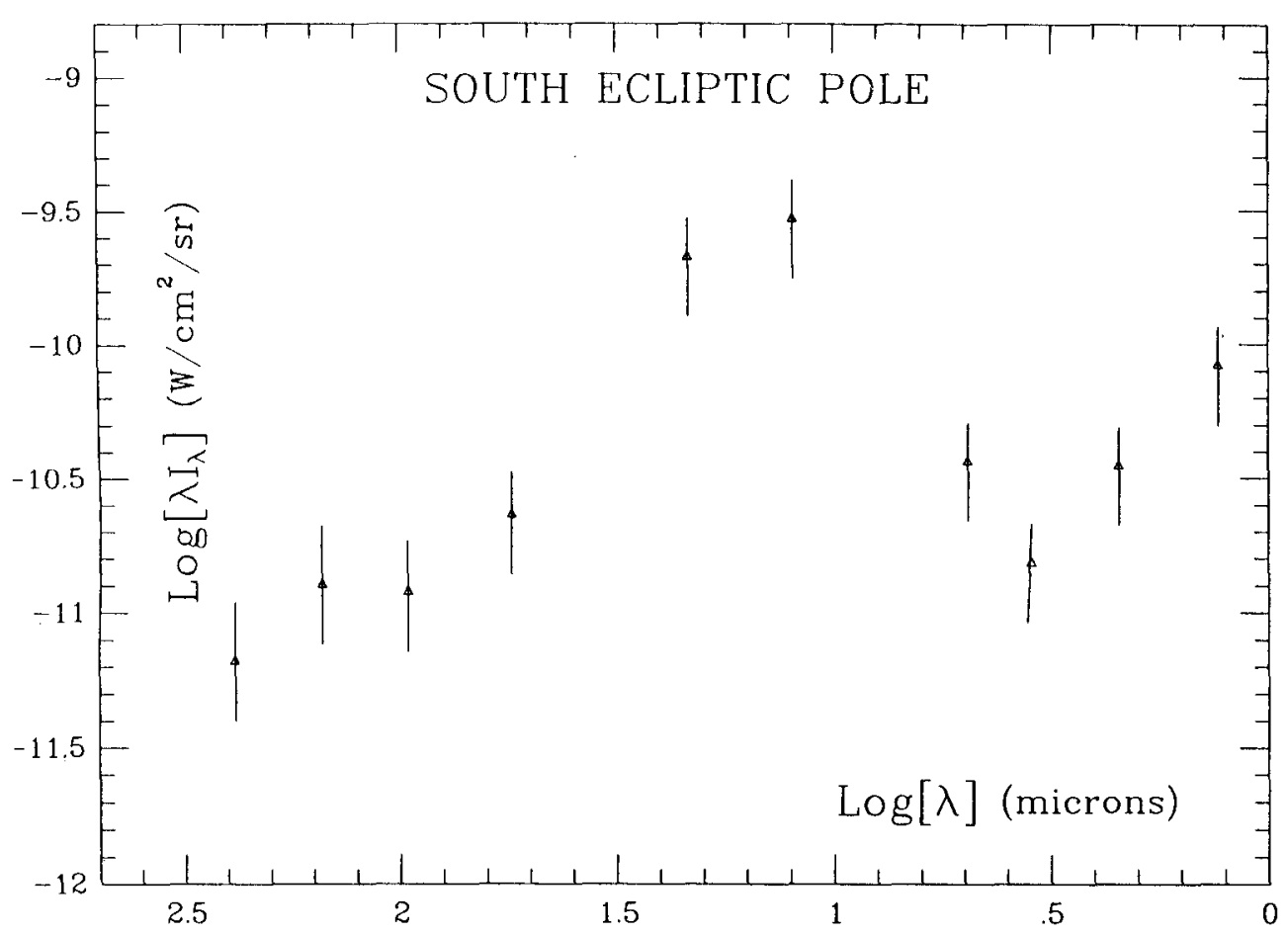

Fig. 3. South Ecliptic Pole brightness measured by DIRBE.

total brightness) at the longest wavelengths.

We also find that the IRAS DC gain at 60 and $100 \mu \mathrm{m}$ is substantially too high (see the IRAS Explanatory Supplement, pp. IV-9 and IV-10 for a discussion of the IRAS DC response). The combined effect of these differences is particularly large at $100 \mu \mathrm{m}$ toward faint sky regions: the IRAS ecliptic pole brightness is nearly 3 times brighter than that reported here. Since the IRAS gain depends on the source angular scale (scan-modulated instrument) and on the source brightness, no simple conversion between the data from the two experiments can be defined.

\section{Summary and Conclusions}

The COBE has mapped nearly the entire sky with two beam sizes at many wavelengths from $1 \mu \mathrm{m}$ to $1 \mathrm{~cm}$. Its performance in orbit meets or exceeds the design goals, and the maps show the expected local sources. The detailed analysis of the data to model and remove these local foreground sources is just begirning, but limits on the cosmic $y<10^{-3}$ and $\mu<10^{-2}(3 \sigma)$ distortions of the $2.735 \pm 0.06 \mathrm{~K}$ background spectrum have been obtained. The dipole anisotropy has the expected shape for a Doppler shifted blackbody as measured by both DM; and FIRAS, and the dipole amplitude and direction are consistent with previors caeasurements. 
The extensive, high-quality DIRBE data promise to allow a very sensitive search for cosmic infrared background radiation.

\section{Acknowledgements}

It is a pleasure to acknowledge the vital contributions of all those at GSFC who devoted their efforts to making this challenging mission not only possible but enjoyable as well. The National Aeronautics and Space Administration/Goddard Space Flight Center (NASA/GSFC) is responsible for the design, development, and operation of the Cosmic Background Explorer (COBE). GSFC is also responsible for the development of the analysis software and for the production of the mission data sets. The COBE program is supported by the Astrophysics Division of NASA's Office of Space Science and Applications.

\section{References}

Bennett, C. L., et al. : 1991, in preparation

Bond, J. R., Carr, B. J., and Hogan, C. J.: 1986, Ap. J. 306, 428-450

Gulkis, S., Lubin, P.M., Meyer, S.S., and Silverberg, R. F.: 1990, Sci. Am. 262(1), 132-139 Mather, J. C.: 1982, Opt. Eng. 21(4), 769-774

Mather, J.C., Cheng, E.S., Eplee, R.E., Jr., Isaacman, R. B., Meyer, S. S., Shafer, R. A., Weiss, R., Wright, E.L., Bennett, C. L, Boggess, N. B., Dwek, E., Gulkis, S., Hauser, M. G., Janssen, M., Kelsall, T., Lubin, P. M., Moseley, S. H. Jr., Murdock, T. L., Silverberg, R. F., Smoot, G. F., and Wilkinson, D. T.: 1990, Ap. J. 354, L37-L41

Matsumoto, T., Hayakawa, S., Matsuo, H., Murakami, H., Sato, S., Lange, A. E., and Richards, P. L.: 1988a, Ap. J. 329, 567-571

Matsumoto, T., Akiba, M., and Murakami, H.: 1988b, Ap. J. 332, 575

Smoot, G. S. et al.: 1990, Ap. J 360, TB 